\title{
Two-segment sural nerve conduction measurements in polyneuropathy
}

\author{
K KAYED, Ø RøSJø \\ From the Laboratory of Clinical Neurophysiology, Akershus Central Hospital, University of Oslo, \\ Nordbyhagen, Norway
}

SUMMARY Sensory conduction velocity of the sural nerve was measured in $\mathbf{5 0}$ normal subjects and 100 consecutive patients with suspected or established diagnosis of peripheral neuropathy. The sensory action potential was recorded by the orthodromic technique for the distal and the proximal segments. In the patient group, sensory conduction of the proximal segment appeared to be more consistant and easier to carry out as compared to the distal segment. Measurements of the latter segment alone could sometimes be misleading. Some practical points acquired from our experience with this method that allow considerable reduction of the examination time and the patients's discomfort are reported.

Many neuropathies begin or are most severe in the lower extremities. Electrical examination of sensory function in the lower limb nerves therefore is mandatory in early, atypical or subclinical cases of polyneuropathy. There is general agreement that of all sensory nerves of the leg, the sural nerve provides the highest diagnostic yield. Although measurements of the sural nerve conduction as a clinical diagnostic procedure is by no means new, ${ }^{1}$ there are considerable differences in the reported normal values, due to variability of the methods by which the sensory action potentials (SAPs) are recorded and measured. The most common technique for routine recording of the sural SAP is the bipolar antidromic method, using surface electrodes, with the latencies calculated from a single sweep or after averaging. In a few laboratories the use of monopolar recording by needle electrodes is the method of choice. Trials to standardise these methods were reported recently by Schuchmann ${ }^{2}$ and Troung and his associates. ${ }^{3}$ Both workers measured fixed segments of the sural nerve as a way to reduce experimental measurement errors.

Address for reprint requests: Dr K Kayed, Akershus Central Hospital, 1474 Nordbyhagen, Norway.

Received 19 February 1983. Accepted 14 April 1983
The monopolar orthodromic technique for recording SAPs was described by Buchthal and Rosenfalk. ${ }^{4}$ The same technique, in a modified form, was applied to the sural nerve. ${ }^{5}$ The same authors reported the normal values of maximum sensory conduction velocities (SCVs) in various nerves including the sural. ${ }^{6}$ This method has been criticised as being too sophisticated in its technical refinements, making it beyond the routine capabilities of many diagnostic units. ${ }^{7}$ With today's rapidly developing electronic technology, most laboratories have the necessary hardware to carry out such procedures without any technical or economic difficulty. This paper reports on orthodromic monopolar recording of the proximal and distal sural SAP in normal subjects, and in patients with various types of polyneuropathies. Some of the practical points aquired from our experience with this method will be discussed.

\section{Material and methods}

The control group included 50 normal subjects between the ages of 25 to 77 years who did not have clinical or neurophysiological findings of polyneuropathy. The patient group included 100 consecutive patients between ages of 25 and 80 years who were referred to our laboratory with the diagnosis of suspected or established 
polyneuropathy. This unselected material represented both the usual and the rare forms of this disease. The most common form was polyneuropathy of alcoholic origin (26). The others were diabetic polyneuropathy (17), peroneal muscular atrophy (18), postinfective (6), intoxications (5), B12 malabsorption (4) and other rare forms (3). The aetiology of the polyneuropathy was unknown in 21 cases.

A Medilec MS6 electromyograph was used. The stimulus was provided by either a constant current or constant voltage generator using surface electrodes. The sensory fibres were stimulated maximally with $2 \mathrm{~Hz}$ pulses of 0.05-0.1 ms duration. The evoked SAP was recorded by DISA type 13L60 teflon coated stainless steel electrodes. For the proximal segment the stimulator was applied behind the lateral malleolus and the recording needles at the junction between the lower and middle third of the leg at a distance of $12-15 \mathrm{~cm}$. For the distal segment the stimulator was applied to the dorsum pedis and the recording needles behind the lateral malleolus. As soon as a satisfactory response appeared on the scope, the averaging was stopped. If necessary, the potentials were scaled up by a factor of two or more for greater ease of measurement, by overriding the automatic scaling of the averager. Calculation of the maximum SCV in these segments were determined from the latency to the first positive peak of the usually triphasic potential. The surface temperature of the extremity was measured by a digital precision thermometer and varied from $35-37^{\circ} \mathrm{C}$. Ambient room temperature was maintained around $23-24^{\circ} \mathrm{C}$. In order to facilitate detection of normal and pathological values of maximum SCV two separate regression lines were plotted for the expected mean values for ages from 20-80 years derived from two formulae given by Buchthal and associates. ${ }^{6}$ For calcula- tion of the proximal segment the formula 57.4-0.05 $\times$ age (SD $3.7 \mathrm{~m} / \mathrm{s}$ ) and for the distal segment 51.8-0.06 $\times$ age (SD $4.6 \mathrm{~m} / \mathrm{s}$ ) were used. Two separate regression lines were also plotted for means values -2 and -2.5 SD. SCV values were considered pathological when they fell below the calculated mean conduction for the patient age -2.5 SD. The values of sural SCV in different types of polyneuropathies were plotted on these two curves (fig 1).

\section{Results}

In the control group the sural SCV was calculated directly by dividing the distance by the latency and was compared to the expected mean normal value for the same age as derived from the regression curves. No significant differences appeared between these two sets of values thus confirming the validity of the formulae for mean measurements in the two segments of the nerve. The mean value for the proximal segment for all $\mathbf{5 0}$ subjects in the proximal segment as calculated directly was $54.3 \pm 4 \mathrm{~m} / \mathrm{s}$ and by the formulae $53.9 \pm 3.7 \mathrm{~m} / \mathrm{s}$. The corresponding values for the distal segment were $49.5 \pm 4.6$ and $50 \cdot 0 \pm 4.5 \mathrm{~m} / \mathrm{s}$.

The figure shows the values of maximum sural SCV in the proximal and distal segments in patients with alcoholic, diabetic, PMA and other types of polyneuropathy combined. It shows that the measurements of the proximal segment are much more stable and easier to record than the distal segment.

Table Summary of previous studies of sural nerve conduction

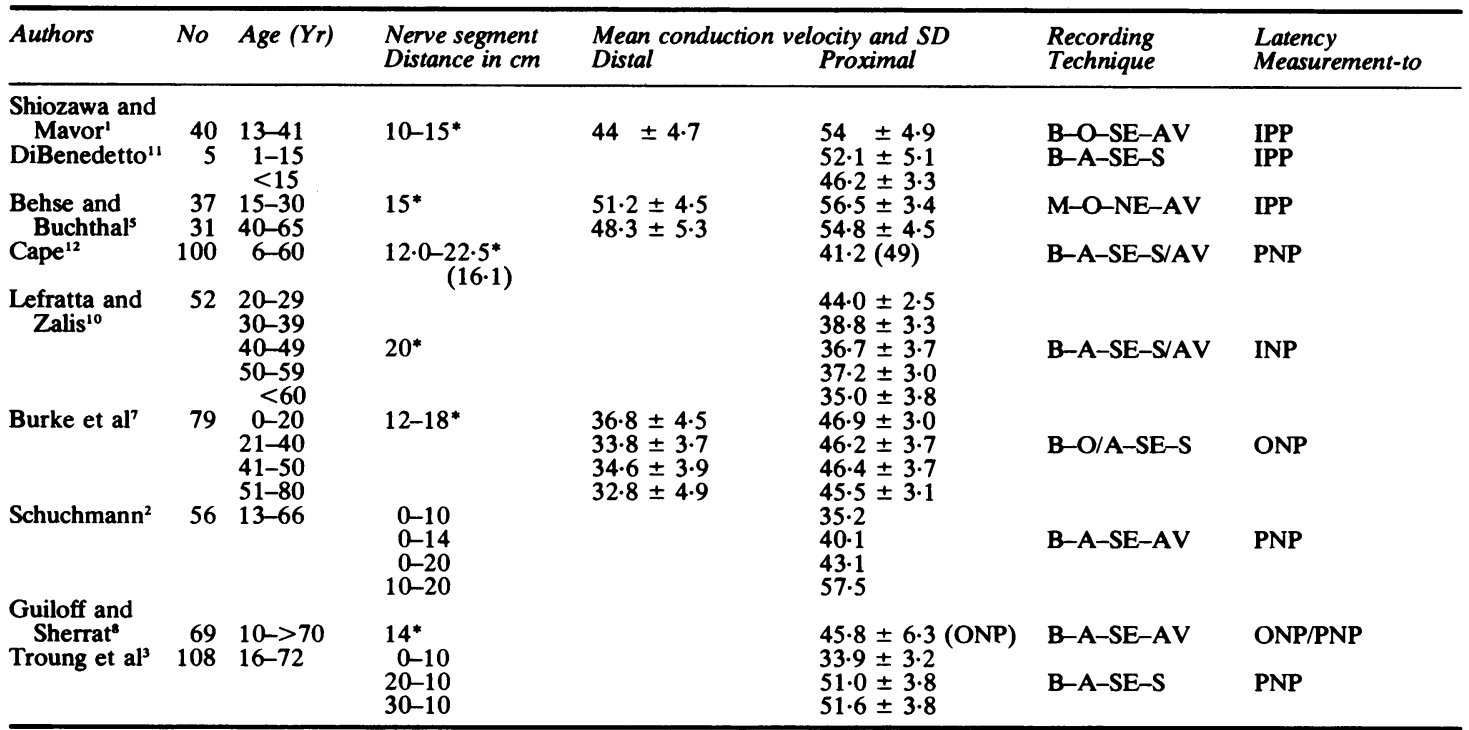

* = Proximal segment, $B=$ Bipolar, $M=$ Monopolar, $\mathbf{S E}=$ Surface electrode, $\mathrm{NE}=\mathrm{Needle}$ electrode, $\mathrm{A}=\mathrm{Antidromic}$, $\mathrm{O}=$ Orthodromic, S = Single, AV = Averaging, IPP = Initial positive peak, PNP = Peak of negative potential, ONP = Onset of negative potential. 



Fig Sural nerve conduction in the proximal and distal segments in various types of polyneuropathy. ( 8 ) Proximal conduction that could not be measured in the distal segment $(\square)$.

The later segment could not be measured in 14 cases. This was especially the case when mean SCV in the proximal segment were around $30 \mathrm{~m} / \mathrm{s}$ or lower. The scatter of the values in the distal segment appeared to be much greater as compared to the proximal one. While measurements of the proximal segments showed pathological values in all patients, few values in distal segments, were within the normal range.

\section{Discussion}

Most authors agree that the sural nerves provide the best index of lower limb sensory function. ${ }^{7}$ However, Guiloff and Sherratt ${ }^{8}$ claimed that the medial plantar SAP was a more sensitive indicator than the sural nerve in 13 cases of polyneuropathy. The same authors reported that the sural nerve action poten- tial amplitude was much greater than the medial plantar SAP. These authors recorded the sural SAP antidromically from the proximal segment and the medial plantar SAP orthodromically from the distal segment. Such comparison, in our opinion, is unwarranted because neither the segment of the nerve nor the recording technique were similar.

Table 1 summarises most of the previous studies of sural nerve conduction. Although some of the values of mean conduction are similar, there appear to be wide variations of normal values given by different authors. In the proximal segment the mean SCV varied from 33.9 to $57.5 \mathrm{~m} / \mathrm{s}$ and in the distal segment from 32.8 to $51.2 \mathrm{~m} / \mathrm{s}$. These variations are mainly due to either the choice of the technique of recording (antidromic vs. orthodromic) or the choice of the method of measurement of the SAP latencies. 
The reason why orthodromic conduction is to be preferred to antidromic conduction is the fact that while antidromic and orthodromic conduction time are identical, the onset of antidromic potential is less well defined than that of the orthodromic potential. Besides, when the threshold of the antidromic potential exceeds that of the muscle action potential the two responses may interfere. ${ }^{4}$ Both factors contribute to measurement errors. Another important reason for these variations is due to the different methods of latency measurements. The landmark commonly used are the initial positive, initial negative or the peak of the negative potential. According to Murai and Sanderson 9 peak latency was longer in antidromic sensory recording as it depended on the distance between the recording electrodes. LaFratta and Zalis ${ }^{10}$ reported that measurements to the onset of the sensory evoked potential added about $5 \%$ to the conduction velocity measured from latencies to the peak of the negative deflection. These authors recommended measuring of the proximal conduction velocity. They found no difference between mid-calf and proximal calf measurements of the sural nerve so that a single segment could be selected. The same authors and Schuchmann ${ }^{2}$ warned against measurements of sensory conduction over short segments of nerves as being liable to measurement errors.

Measurement errors of the distal segment can explain the wide range of scatter of SCV values in this relatively short segment. Also it can explain the paradoxical finding that while all the proximal segment values were pathological, few of the distal values appeared to be within the normal range, despite the fact that polyneuropathy ususally starts or is more severe distally.

Routine amplitude measurements of the sural SAPs were not carried out. This was due to the fact that reproducibility of amplitude measurements require at least $2-3$ successive averaging runs thus increasing both the examination time as well as the patient's discomfort. Most of our patients while easily tolerating needle electrode punctures, did complain when repetitive stimulation continued for longer periods. The aim was to make the procedure more agreeable and acceptable to the patient, especially when future control examinations were to be considered.
The results of this study suggest that proximal sural nerve sensory conduction when measured by the orthodromic monopolar technique, according to the above mentioned guidelines, could provide a practical standardised method of examination of sensory function of the lower limbs in polyneuropathy. In our experience measurements limited to the distal segment could sometimes be misleading. Two segment measurements doubles both the examination time and the patient's discomfort and did not seem to provide relevant additional data to those derived from measurements of the proximal segment alone.

\section{References}

' Shiozawa R, Mavor H. In vivo human sural nerve action potentials. J Appl Physiol 1969;26:623-9.

${ }^{2}$ Schuchmann JA. Sural nerve conduction: standardized technique. Arch Phys Med Rehabil 1977;58:166-8.

${ }^{3}$ Truong XT, Russo FJ, Vagi I, Rippel DV. Conduction velocity in the proximal sural nerve. Arch Phys Med Rehabil 1979;60:304-8.

${ }^{4}$ Buchthal F, Rosenfalk A. Evoked action potentials and conduction velocity in human sensory nerves. Brain Res 1966;3:1-122.

${ }^{5}$ Behse F, Buchthal F. Normal sensory conduction in the nerves of the leg in man. J Neurol Neurosurg Psychiatry 1971;34:404-14.

${ }^{6}$ Buchthal F, Rosenfalk A, Behse F. Sensory potentials of normal and diseased nerves. In: Dyck PJ, Thomas PK, Lambert EH, eds. Peripheral Neuropathy. Philadelphia: WB Saunders 1975; vol. 1:442-64.

${ }^{7}$ Burke D, Skuse NF, Lethlean AK. Sensory conduction of the sural nerve in polyneuropathy. $J$ Neurol Neurosurg Psychiatry 1974;37:647-52.

${ }^{8}$ Guiloff R, Sherratt RM. Sensory conduction in medial plantar nerve. J Neurol Neurosurg Psychiatry 1977;40:1168-81.

${ }^{9}$ Murai Y, Sanderson I. Studies of sensory conductions. Comparison of latencies of orthodromic and antidromic sensory potentials. J Neurol Neurosurg Psychiatry 1975;38:1187-9.

${ }^{10}$ LaFratta CW, Zalis AW. Age effects on sural nerve conduction velocity. Arch Phys Med Rehabil 1973;54: 475-7.

"DiBenedetto M. Sensory nerve conduction in lower extremities. Arch Phys Med Rehabil 1970;51:253-8.

${ }^{12}$ Cape CA. Sensory nerve potentials of peroneal, sural and tibial nerves. Am J Phys Med 1971;50:220-9. 